\title{
Semantic feature production norms for a large set of living and nonliving things
}

\author{
KEN MCRAE \\ University of Western Ontario, London, Ontario, Canada \\ GEORGE S. CREE \\ University of Toronto, Scarborough, Ontario, Canada \\ MARK S. SEIDENBERG \\ University of Wisconsin, Madison, Wisconsin \\ and \\ CHRIS MCNORGAN \\ University of Western Ontario, London, Ontario, Canada
}

\begin{abstract}
Semantic features have provided insight into numerous behavioral phenomena concerning concepts, categorization, and semantic memory in adults, children, and neuropsychological populations. Numerous theories and models in these areas are based on representations and computations involving semantic features. Consequently, empirically derived semantic feature production norms have played, and continue to play, a highly useful role in these domains. This article describes a set of feature norms collected from approximately 725 participants for 541 living ( $\mathrm{dog}$ ) and nonliving (chair) basic-level concepts, the largest such set of norms developed to date. This article describes the norms and numerous statistics associated with them. Our aim is to make these norms available to facilitate other research, while obviating the need to repeat the labor-intensive methods involved in collecting and analyzing such norms. The full set of norms may be downloaded from www.psychonomic.org/archive.
\end{abstract}

In many of the most influential theories of word meaning and of concepts and categorization, semantic features have been used as their representational currency. For example, classical, prototype, and exemplar theories of categorization and conceptual representation all make use of features (Medin \& Schaffer, 1978; Minda \& Smith, 2002; Smith \& Medin, 1981), as do network models of semantic memory and language processing (Collins \& Loftus, 1975). Numerous vector models of memory also are based on featural representations (Hintzman, 1986; Murdock, 1982), as are connectionist models of object recognition (Plaut, 2002), word recognition (Harm \& Seidenberg, 2004), and semantic memory (Hinton \& Shallice, 1991; Plaut \& Shallice, 1993).

This work was supported by Natural Sciences and Engineering Research Council Grant OGP0155704 and NIH Grants R01-DC0418 and R01-MH60517 to K.M., an Ontario Graduate Scholarship and a Natural Sciences and Engineering Council Doctoral Fellowship to G.S.C., Grants NICHD 29891 and NIMH K02-01188 to M.S.S., and a SharcNet Graduate Student Fellowship to C.M. When using these norms, please refer both to this article and to the grants that supported it. Correspondence concerning this article should be addressed to K. McRae, Department of Psychology, Social Science Centre, University of Western Ontario, London, ON, N6A 5C2 Canada (e-mail: mcrae@uwo.ca).

Note-This article was accepted by the previous editor, Jonathan Vaughan.
The major purpose of collecting semantic feature production norms is to construct empirically derived conceptual representations that can be used to test theories of semantic representation and computation. Typically, participants are presented with a set of concept names and are asked to produce the features they think are important for each concept. The representations of moose and knife are presented in Appendix A as examples of the types of representations derived from such norms, which typically include a list of features for a concept, together with production frequency, the number of participants who listed each feature for the concept. This basic information can then be extended with various statistical analyses, supplemented with additional measures, and used to test predictions with both human experiments and computational modeling. The goal of the present article is to describe a large set of semantic feature production norms that have been collected for 541 living and nonliving thing concepts, easily the largest such set in existence, and to make these norms publicly accessible. In the remainder of this article, we briefly describe the uses of semantic feature norms, discuss their strengths and weaknesses, and then describe our norms and the accompanying measures and statistics we have made available.

\section{Utility of Feature Norms}

Given the importance of semantic features in shaping theories, researchers have recognized the value of collect- 
ing empirically based semantic feature production norms for testing hypotheses, constructing experimental stimuli, and generating representations for implemented models. To the best of our knowledge, although several normative databases currently exist, only one set is publicly accessible (Garrard, Lambon Ralph, Hodges, \& Patterson, 2001; 64 living and nonliving things).

Other sets of norms have been collected and used for various purposes, although the norms themselves have not been published. For example, Rosch and Mervis (1975) collected norms for 20 basic-level concepts from each of six superordinate categories and used them to explore typicality gradients. Ashcraft (1978b) collected norms for 140 living and nonliving things to use primarily for constructing feature verification experiments. Hampton (1979) collected features for eight superordinate categories and used them to test Smith, Shoben, and Rips's (1974) model of category verification and to predict verification latencies. Wu and Barsalou (2004) used feature norms to compare predictions derived from theories based on perceptual symbol systems versus amodal semantics. Devlin, Gonnerman, Andersen, and Seidenberg (1998; 60 living and nonliving things) and Moss, Tyler, and Devlin (2002; 93 living and nonliving things), like Garrard et al. (2001), used their norms to investigate accounts of categoryspecific semantic deficits. Vinson and Vigliocco (2002) collected norms for 230 nouns and 216 action verbs and used them to compare computations underlying nouns versus verbs in a number of experimental paradigms. In addition to using their norms for analyzing the structure of conceptual representations and designing behavioral experiments, Devlin et al., Moss et al., and Vinson and Vigliocco used them to derive representations for implemented computational models.

In addition, feature norms and featural representations derived from them have served as the basis of accounts of numerous empirical phenomena, such as semantic similarity priming (Cree, McRae, \& McNorgan, 1999; McRae, de Sa, \& Seidenberg, 1997; Vigliocco, Vinson, Lewis, \& Garrett, 2004), feature verification (Ashcraft, 1978a; McRae, Cree, Westmacott, \& de Sa, 1999; Solomon \& Barsalou, 2001), categorization (Hampton, 1979; Smith et al., 1974), and conceptual combination (Hampton, 1997; Smith, Osherson, Rips, \& Keane, 1988), and have been used to provide support for modality-specific aspects of representation (Pecher, Zeelenberg, \& Barsalou, 2003; Solomon \& Barsalou, 2001).

Although the vast majority of feature-based studies have investigated the representations of concrete nouns, feature norms have also been used successfully to provide insight into the nature of verb representation. For example, Vigliocco et al. (2004) have used both noun and verb representations based on feature norms to predict the likelihood that a noun or a verb will be substituted for a semantically related concept, using a picture-naming task to induce substitution errors, and to predict the amount of interference of a distractor word in a picture-word interference task. In a somewhat different application, McRae,
Ferretti, and Amyote (1997) used feature norms to gain insight into the types of conceptual information people possess for thematic roles of verbs (agents and patients, in this case). In summary, featural representations have been used to provide insight into a number of phenomena involving semantic memory and categorization.

\section{Distributional Statistics Derived From Feature Norms}

Numerous measures and distributional statistics can be calculated from the norms, such as estimates of feature saliency (production frequency, cue validity) and measures of how features of various types are distributed across concepts (number of color or taste features). Several measures can also be used to augment the information obtained from the norms themselves, such as concept familiarity ratings, word frequency counts, and orthographic and phonological variables associated with a concept's name. These and other variables, which are described more fully below, can be used both to manipulate a variable between stimulus groups and to equate stimuli on nuisance variables with increased confidence. For example, because of the norms, McRae et al. (1999) were able to study the influence of feature correlations on the computation of word meaning while equating for nine potentially confounding variables, a level of control that would have been impossible without the norms.

Distributional statistics, such as statistical regularities among features, have proven particularly insightful with regard to understanding semantic computation. For example, McRae, de Sa, and Seidenberg (1997; McRae et al., 1999) used the norms to illustrate the role of feature correlations in semantic computations. Pexman, Holyk, and Monfils (2003) and Pexman, Lupker, and Hino (2002) used our norms to show that the number of features listed for each concept predicts aspects of semantic processing, including reading time when the concept name is presented in a moderately congruent sentence context.

Cree and McRae (2003) have presented the most detailed distributional analyses of feature norms to date, including the salience of information types carried by various features according to a knowledge type taxonomy that was designed to be linked to neural processing substrates, as well as numerous distributional statistics that have been implicated as important aspects of semantic representations and computations. These analyses provide insight into the behavioral phenomena underlying category-specific semantic deficits, and the statistics upon which they were based are included in the downloadable norms.

Featural representations derived from production norms are also useful for computational modeling that requires vector representations, because such representations can be derived easily from norms. Cree et al. (1999) argued that a major advantage of basing models on empirically derived representations, rather than on either algorithmically generated pseudorandom representations or representations based on experimenters' intuitions, is that they substantially reduce degrees of freedom in modeling. That 
is, the representations are determined by the participants in the norming task, rather than by the modeler. Perhaps even more critical for learning models such as connectionist networks, having reasonably valid distributional statistics of the patterns to be learned can have important and beneficial consequences. That is, accurately simulating human behavior on relevant tasks requires training a model on representations that closely approximate the actual statistics in the world. This is difficult to guarantee in models that are based on pseudorepresentations that may capture only broad generalities of the underlying distributional statistics. This point was made clearly in the past tense verb modeling of Daugherty and Seidenberg (1992), in which simulations were successful only when the model was trained on patterns that exemplified the proper distributional information.

\section{Scope and Limits of Feature Norms}

Feature norms are assumed to provide valid information not because they yield a literal record of semantic representations (i.e., they do not provide a verbatim readout), but rather because such representations are used systematically by participants when generating features (Barsalou, 2003). As we have stated previously, we do not believe that semantic knowledge is represented in the brain literally as a list of verbalizable features. Rather, when participants produce features in a norming task, they directly exploit representations that have developed through repeated multisensory exposure to, and interactions with, exemplars of the target category. Barsalou used a perceptual symbol systems framework to describe processes underlying feature production. He stated that when participants generate features, they construct a holistic simulation of the target category, then interpret this simulation by using featural and relation simulators. Rather than being a measure of a category's underlying static memory abstraction (which we agree does not exist), a participant's list of features represents a temporary abstraction that is constructed online for the purpose of producing feature names. Therefore, the dynamic nature of feature listing results in substantial variability both across and within participants. To deal with this variability, we had numerous participants list features for each concept (30 per concept in our norms) and then pooled responses to derive a single, averaged representation. Thus, feature listing provides a window into important aspects of word meaning, without necessarily being definitive (Medin, 1989).

One limitation of feature norms is that they are linguistically based. Because people convey their conceptual knowledge through a linguistic filter, some types of information are transmitted more clearly than others. For example, information types such as parts ( $<$ has a handle $>$ ), color $(<$ is red $>$ ), statements about what an object typically is used for ( $<$ used for cutting $>$ ), who typically uses it ( $<$ used by children $>$ ), where an object or entity typically is located ( $<$ found in kitchens $>$ ), and characteristic behaviors of animals ( $<$ barks $>$ ) appear to be relatively easily verbalizable. However, some types of knowledge that almost certainly play an important role in capturing various behavioral phenomena are omitted to a large extent in verbal feature norms, particularly when participants must produce features as short written descriptors. For example, for many visually based tasks, information about the spatial relations and size relations among parts is integral to recognizing objects and entities. Because these types of knowledge are quite difficult to verbalize, they simply are missing from the norms (see Cree \& McRae, 2003, for further discussion). Another example pertains to recognition of animals as they naturally move about. Knowledge of how, for example, cats, dogs, rabbits, and squirrels move helps people recognize them, particularly from a distance, but this type of information is difficult to verbalize and so is not well-represented in the norms (although information concerning animals that have a relatively distinguishing type of movement that can be verbalized, such as $<$ hops $>,<$ swims $>$, or $<$ runs fast $>$, are found in the norms). Of course, if valid representations of these types of information become available, they could be used to augment the norms.

Any task such as feature listing carries with it certain demand characteristics for participants. One fact that becomes obvious when feature norms are analyzed is that participants' responses are somewhat biased toward information that distinguishes among concepts - that is, the pieces of information that enable people to distinguish a concept from other, similar concepts. Participants appear to either interpret this as a primary component of their task when listing features, or alternatively, this is the type of information that is highly salient to people. Of course, distinguishing information is important to determining category membership and similarity among concepts (Tversky, 1977). In addition, it may be the case that this type of information has a privileged status in conceptual representations (Cree \& McRae, 2003; McRae, Cree, Cho, \& McNorgan, 2003). As a consequence of this tendency to list distinguishing features, participants in semantic feature production tasks tend to list few features that are true of large numbers of entities or objects, such as $<$ has a heart $>$, particularly those that are internal and not particularly salient (although they do tend to list internal properties of fruits, vegetables, and foods, presumably because they are salient, given that we open them up and eat them; see Cree \& McRae, 2003). Although occasionally interpreted as a weakness of such norms, it may actually be a strength, because it appears that these general features play only a small role in object identification, language comprehension, and language production precisely because they are not salient and are true of large numbers of concepts.

One other aspect of verbally produced features is that they vary widely in the complexity of the knowledge for which they stand as placeholders. For example, color features, such as $<$ is green $>$ for apple, or parts, such as $<$ has four wheels $>$ for car, appear to be relatively simple 
and straightforward. In contrast, many functional features, such as <used for carpentry $>$ for hammer, likely are stand-ins for a more complex array of situational/ functional knowledge regarding the conditions under which a hammer is used (Barsalou, Sloman, \& Chaigneau, 2005).

Research with human participants has revealed that representations based on feature norms are probably best suited to capturing performance on certain types of tasks - namely, online recognition or semantic decision tasks, many of which use words as stimuli (Jones \& Smith, 1993; McRae, de Sa, \& Seidenberg, 1997). There are, however, different levels at which conceptual representation can be studied. For example, knowledge-based theories of concepts stress somewhat different types of knowledge and tasks. Paradigms in which participants are required to reason deeply about concepts - such as deciding, for example, whether a cat that has an operation to make it look like a skunk is still a cat or not-require knowledge that is most likely outside the scope of feature norms (Keil, 1989). Much of the research conducted under the guise of knowledge-based theories has focused on explicit knowledge of relations among features, particularly causal or dependency relations (Medin \& Shoben, 1988). Feature norms do not include this type of knowledge. They do provide the basic features, however, and implicit statistical knowledge regarding how features are correlated can be computed from them. Therefore, the norms may provide insight into phenomena regarding features relations (vs. correlations). For example, McNorgan, Kotack, Meehan, and McRae (in press) found that the magnitude of the correlation between two features predicts people's explicit ratings of the degree to which features are related. In addition, Rogers and McClelland (2004) have outlined feature-based explanations for a number of phenomena that have been cited as evidence for knowledge-based theories. In summary, although knowledge-based theories stress what might be termed higher order knowledge, they do include featural representations, and people's theories presumably interact with (or arise in part from) distributional statistics that can be estimated from norms (McRae, 2004).

In defending the need for knowledge-based theories, it has been argued that feature-based theories are limited in their utility because they are too unconstrained (Medin, 1989). A major argument has been that there are an infinite number of features that could potentially be listed for any particular concept. For example, a dog can be described as being $<$ larger than a pencil $>,<$ slower than a jet $>,<$ rarely found in office buildings $>$, and so forth. We believe, however, that the use of empirically grounded feature norms deals with the concerns that the number of possible features is virtually unlimited and that features are unconstrained. For example, features such as $<$ is larger than a tulip $>$ or $<$ moves faster than an infant $>$, although logically possible, do not occur in norms. The computations described above that are assumed to underlie performance in a feature production task do not lead to the production of these sorts of features. Although people are capable of verifying that a $d o g<$ is larger than a pencil $>$ and could probably even produce such features, given sufficient explicit guidance to do so, these sorts of statements are not generated as a result of running a perceptual simulation (Barsalou, 1999) or accessing core information underlying a concept (Hampton, 1979). No such features were produced by any of the approximately 725 participants who listed features in our norming task.

In summary, although the emergence of knowledgebased theories resulted in a period of disenchantment with feature norms and feature-based theories for some researchers, interest in semantic features has increased recently because of a large body of behavioral and modeling studies in which such features play an explanatory role in providing insight into semantic computations. Simply put, measures based on feature norms have been used to account for a great deal of behavioral phenomena.

\section{THE NORMS}

\section{Stimuli}

Each of the 541 normed concepts corresponds to a single English noun. They were chosen to cover those used in various experiments regarding semantic memory tasks in investigations of both normal adults and neuropsychological patients. An initial set of 190 concepts, reported in McRae, de Sa, and Seidenberg (1997), was chosen from Battig and Montague (1969) and Rosch and Mervis (1975). The remaining concepts were chosen by combing the literature for relevant articles in which items were reported. This set was compiled with the assistance of Matt Lambon Ralph, James McClelland, Karalyn Patterson, and Tim Rogers. Thus, the 541 concepts cover a broad range of living and nonliving things used in previous studies. They also were chosen to span a wide range of familiarity, although of course, participants must be reasonably familiar with both the concept and its name to be able to produce useful information.

We attempted to avoid ambiguous concept names, although this is impossible given the fact that such a large proportion of English words are ambiguous. Noun-verb ambiguities were dealt with by instructing the participants to provide features for the noun meaning of the word (e.g., hammer as an object, rather than the verb meaning of hammer), and responses showed that this was successful. In terms of noun-noun ambiguities, for the vast majority of cases in which we identified the ambiguity a priori, a cue was provided to disambiguate the noun. For example, some participants listed features for bat (baseball), whereas others listed features for bat (animal). In our experience, if an ambiguity exists, at least some participants will make reference to it. For example, when providing features for anchor, a few participants listed $<$ reads the news on television $>$. However, very few participants listed features for a meaning of the word that was either quite low in familiarity or colloquial; for example, the participants did not list features regarding messy, slovenly people for pig. We are currently entering norms that have been collected for approximately 200 additional concepts, 
thus further increasing both the breadth and the depth of coverage. These will be added to the database when they are completed.

\section{Collection}

The norms have been collected in three main phases since 1990. In the first phase, norms for 190 basic-level concepts were collected (10 superordinate concepts were also included in these norms, although they are not discussed further herein). Initially, 500 forms were distributed in McGill University psychology classes, and 167 were returned (33\%). In the second round, 200 forms were distributed, and 80 were returned (40\%). The remainder of the forms were collected in the laboratory. Each participant in the first phase listed features for 20 concepts. Second, norms for 76 concepts were collected at the University of Southern California. Participants listed features for 24 concepts, and these were collected in class. The remaining norms (288 concepts) were collected over a period of 3 years at the University of Western Ontario. This phase was conducted either in groups or individually in the laboratory, using forms that were identical to those used in the second phase. Note that a few concepts have been discarded either because of the participants' lack of familiarity with the target category (e.g., heron) or because responses were split between alternative meanings of a word (e.g., file: nail file vs. wood file vs. collection of papers; this was part of the initial set of 190 concepts, in which disambiguating cues were not used).

For all of the norms, the participants were provided with 10 blank lines to list features. The participants were asked to list features of the things to which the words referred. They were asked to list different types of features, such as physical (perceptual) properties (how it looks, sounds, smells, feels, and tastes), functional properties (what it is used for and where and when it is used), and other facts about it, such as the category it belongs in or other encyclopedic facts (such as where it is from). Three examples were provided. Sample instructions are presented in Appendix B. In all cases, 30 participants listed features for each concept. The participants were given as much time as needed; they took approximately $40-50 \mathrm{~min}$.

In all phases, care was taken to avoid having the participants produce features for multiple semantically similar concepts, to avoid explicit comparisons among similar concepts. Each participant produced features for, at most, two concepts that we intuitively considered to be similar, and the corresponding concept names appeared on separate pages of a norming form.

\section{Recording}

For each concept, each feature was recorded with its production frequency, which is the number of participants who listed that feature for that concept (ranging from 1 to 30). A major issue in recording the features was to ensure that synonymous features were recorded identically, both within and among concepts. For example, "used for transportation," "used for transport," "is used for trans- portation," "people use it for transportation," and "transportation" were coded as < used for transportation $>$. It was equally critical to ensure that features that differed in meaning were given distinct labels. Responses were interpreted conservatively, and the validity of all but the most obvious interpretations was verified by multiple colleagues. Note that we did not alter feature names to avoid potential ambiguity. We chose not to, because it was unclear where to draw the line. For example, it does seem safe to assume that $<$ has a trunk $>$ has different meanings for elephant than for car. Furthermore, $<$ has legs $>$ seems to refer to different things for table than for $d o g$ and perhaps should be differentiated. However, it is less clear whether $<$ has legs $>$ should be differentiated for $d o g$ versus canary or grasshopper. Are they sufficiently different types of legs? On what criteria would these decisions be based? In addition, a brick is a different color of red than is a raspberry. Should $<$ is red $>$ be differentiated for these concepts? Finally, a feature such as $<$ is large $>$ perhaps means something different for ostrich than for yacht and, thus, could also be differentiated. Because we wanted to be as conservative as possible, and because these differences in feature meanings form a continuum, so that it is unclear where to draw a line regarding when to form two or more features versus when to leave them alone, we did not change any of them. Of course, users of the norms could sort the concepts file on feature name and make their own judgments, but we have not done so.

The following method was used for interpreting and organizing the feature set. In all cases, quantifiers such as "generally," "usually," and "can be" were dropped from the participants' responses. We assumed that the information provided by these quantifiers would be carried by production frequency; that is, the number of participants including a feature should vary according to how often the instances have the feature or the salience of that feature for the average person. If a participant listed an adjective-noun feature $(<$ has four wheels $>$ ), it was divided (into $<$ has wheels $>$ and $<$ has four wheels $>$ ), under the assumption that the participant had provided two bits of information. Disjunctive features (e.g., $<$ is green or red $>$ ) were also divided $(<$ is green $>$ and $<$ is red $>$ ).

A number of key words and phrases were used to organize and code the features. Examples of these are displayed in italics, with an example completion in normal font: $<a$ vegetable $>$ (superordinate category), $<e g$-jeans $>$, $<$ beh-swims $>$ (beh is an abbreviation for behavior), $<$ inbeh-ticks $>$ (inbeh is an abbreviation for something an inanimate object does seemingly on its own), <bought/ sold in hardware stores $>,<$ causes gas $>,<$ found in kitchens $>$, < $<$ grows underground $>,<$ has paws $>,<$ is brown $>,<$ lives in forests $>,<$ made of metal $>,<$ part of a table setting $>,<$ requires a driver $>,<$ runs on gasoline $>$, <eaten for dessert $>$, <used for carpentry $>$, and $<$ worn by women $>$. The intuitive purpose of using these key words and phrases was to signal feature type. That is, they were used as a means of classifying features into basic types. A number of years after the original norms 
were collected, Wu and Barsalou (2004) developed a taxonomy for classifying features into basic knowledge types in a detailed and sophisticated manner. We revised their taxonomy somewhat for our purposes, and this is described below. Recently, Cree and McRae (2003) developed a brain region taxonomy that incorporated insights from neuroscience and neuropsychology to determine a set of types of knowledge that plausibly may be computed in at least somewhat distinct brain areas. This taxonomy is also described below. All the features in the available norms files are categorized according to these taxonomies.

\section{Measures and Statistics}

In this section, we describe the measures we have collected for the norms and the statistics that we have calculated from them. The following sections describe the Excel files that can be downloaded from the Web site. There are four main files: one containing information regarding individual concepts, one with information regarding the features in general, one with information regarding the features for each concept, and one containing all pairwise similarities among the concepts.

Concepts. The concept-level measures for the 541 concepts are presented in CONCS_brm.xls. The column names from that file and a short explanation of them is presented in Appendix C. The first four columns are the name of each concept, its pronunciation according to the Neighborhood Watch program (Davis, 2005) word frequency from Kučera and Francis (1967), where each concept's name frequency was summed over its singular and plural uses, and the natural logarithm of this frequency. Next is word frequency from the British National Corpus (Burnard, 2000), again summed over its singular and plural uses, and the natural logarithm of it. Familiarity ratings for each concept follow, where 20 participants rated the degree to which they were "familiar with the thing to which the word refers" on a scale of 1 to 9 , with 1 corresponding to not at all familiar and 9 to extremely familiar. Next are physical characteristics of the concept names: number of letters, number of phonemes, number of syllables, mean position-specific bigram and trigram frequencies, as well as Coltheart's $N$ (the number of words that can be created by changing one letter in the concept name). The latter three measures were computed using Davis's Neighborhood Watch program.

From the norms, we calculated a number of measures to describe the distribution of features for each concept. The first is the total number of features listed for the concept by at least 5 of the 30 norming participants, the cutoff we have used to include features. We have also included the same variable, but excluding all taxonomic features. Note that the following variables were also calculated with taxonomic features excluded because we consider statements regarding the superordinate category (or categories) to which a concept belongs as being somewhat different from information regarding other types of features (parts, function, etc.). The taxonomics also include some subordinates and coordinates but are dominated by superordinates.
The next four variables are measures of feature informativeness with regard to specific concepts. Distinguishing features are considered to be critical for discriminating among similar concepts. For example, $<$ moos $>$ distinguishes cows from other, similar animals (and in this case, from all other things), whereas $<$ eats $>$ does not, because many things eat. Distinguishing features have been a part of accounts of a number of empirical phenomena regarding normal participants' performance in typicality judgments (Rosch \& Mervis, 1975), similarity judgments (Tversky, 1977), feature verification (McRae et al., 2003), and category verification (Smith et al., 1974) and also have been important for understanding data regarding category-specific deficits (Cree \& McRae, 2003; Garrard et al., 2001). The number of distinguishing features per concept was calculated as the number of a concept's features that occur in only one or two concepts in the norms. We also calculated the percentage of a concept's features that were identified as distinguishing under this definition (number of distinguishing features divided by the number of features). Distinctiveness (also known as informativeness) is a related measure that reflects the continuum from truly distinguishing to highly shared (Devlin et al., 1998; Garrard et al., 2001). It was calculated for each feature as the inverse of the number of concepts in which the feature appears in the norms, and then mean distinctiveness of each concept's features was calculated and presented. In concert with Devlin et al., but in contrast to Garrard et al., we calculated distinctiveness across all the concepts in the norms, rather than only across concepts within a category. The major reason for this decision is that numerous concepts are in multiple categories, whereas some basic-level concepts do not appear to belong to any psychologically real superordinate category. The final measure of informativeness is cue validity (Bourne \& Restle, 1959; Rosch \& Mervis, 1975). Cue validity is the conditional probability of a concept, given a feature, $P\left(\mathrm{C}_{j} \mid \mathrm{F}_{i}\right)$, which is measured by the probability that a feature will appear in a concept $P\left(\mathrm{~F}_{i} \mid \mathrm{C}_{j}\right)$, divided by the probability that that feature will appear in all relevant concepts, as in Equation 1:

$$
P\left(\mathrm{C}_{j} \mid \mathrm{F}_{i}\right)=\frac{P\left(\mathrm{~F}_{i} \mid \mathrm{C}_{j}\right)}{\sum P\left(\mathrm{~F}_{i} \mid \mathrm{C}_{k}\right)} .
$$

In Equation $1, \mathrm{~F}_{i}=$ feature $i, \mathrm{C}_{j}=$ concept $j$, and $\mathrm{C}_{k}=k$ th concept in the set. Thus, cue validity was calculated as the production frequency of a feature for that particular concept, divided by the sum of the production frequencies of that feature for all concepts in which it occurs in the norms. In the extreme case, if a feature is truly distinguishing, as $<\operatorname{moos}>$ is for $c o w$, it will have the maximum score for distinctiveness and cue validity, which is 1.0. In contrast, if a feature is shared by many concepts, its distinctiveness and cue validity are extremely low (e.g., <eats $>$ for any concept in which it occurs). The concepts file contains the mean cue validity of each concept's features.

Because our interest in a number of projects has been the distributional structure of semantic space (i.e., statistical regularities among features, feature correlations), 
we calculated three variables on the basis of the correlational structure of the feature representations. To do so, we constructed a matrix of the concept representations. The full matrix has 541 rows, 1 for each concept, and 2,526 columns, 1 for each feature. Each matrix element corresponds to the production frequency of a feature for a concept. However, to avoid spurious correlations when the feature correlations were computed, only features that appeared in three or more concepts were included in the correlational analyses. Therefore, to compute feature correlations, we calculated the Pearson product moment correlation between each pairwise combination of the resulting 484 feature vectors. A feature pair was counted as significantly correlated if the features shared at least $6.5 \%$ of their variance. From these data, we calculated three variables that provide an indication of the degree to which a concept's features are intercorrelated. The first is intercorrelational density, which is the sum of the percentage of shared variance across all of a concept's significantly correlated feature pairs (because it is a sum, it is no longer truly a percentage). The next two are the number and percentage of significantly correlated feature pairs per concept. The latter was calculated as the number of significantly correlated feature pairs, divided by the number of pairs of features that occurred in three or more concepts. Note that both criteria, three or more concepts per feature and $6.5 \%$ of the variance, are somewhat arbitrary (although the resulting statistics have proven to have predictive power).

The final set of columns provides estimates of the salience of each brain region knowledge type for each concept (Cree \& McRae, 2003). All the features were classified into one of nine knowledge types: three corresponding to visual information (visual-color, visualparts and surface properties, and visual-motion), four corresponding to other primary sensory processing channels (smell, sound, tactile, and taste), one corresponding to functional/motor information regarding the ways in which people interact with objects (function), and one (encyclopedic) corresponding to all other knowledge types. Although these nine knowledge types are presumably not the only ones that may be processed in relatively distinct neural regions, they are the ones that Cree and McRae felt comfortable positing, given the current state of knowledge. In addition, other types of information may also be important (such as emotional reactions to objects that might be processed in the limbic system), but due to insufficient numbers of these, Cree and McRae excluded this knowledge type from their analyses, classifying them as encyclopedic features instead. Each of the final set of columns in the concepts file contains the number of features of each of these knowledge types for each concept.

Features. The features file, FEATS_brm.xls, contains a row for each of the 2,526 features in the norms. The name and a short description of each column is presented in Appendix D. The first column is the feature name, which is a phrase that captures the meaning of the feature. The second is the number of concepts in the norms in which that feature oc- curs. The third column designates the binary distinguishing/ nondistinguishing variable described earlier, and the next is the feature's distinctiveness. The number of characters in the feature name follows, with each space counted as a character. Finally, the type of each feature according to the brain region taxonomy is included.

Conceptual representations. The CONCS_FEATS_ concstats_brm.xls file contains the featural representation of each concept and associated information about the concepts, features, and the features with respect to each concept. The column names and short descriptions are presented in Appendix E. The concept name is first, then the feature name. Following that is the type of each feature according to the slightly modified $\mathrm{Wu}$ and Barsalou (2004) feature type taxonomy that is described in Appendix F. In this taxonomy, features are classified into one of four major types and then into subtypes; there is a column designating major and minor feature type. The next column is the feature type according to Cree and McRae's (2003) brain region taxonomy. Following that is the production frequency for each feature for each concept- the number of participants out of 30 who listed that feature for that concept. We also calculated the ranked production frequency - that is, the rank of each feature with respect to the other features in a particular concept in terms of production frequency. The number of concepts in which each feature occurred in the norms, along with its inverse (distinctiveness), follows. Cue validity of each feature for each concept is next. In terms of feature correlation statistics, we have included intercorrelational strength of a feature for a concept, calculated both including taxonomic features and excluding them (McRae et al., 1999; McRae, de Sa, and Seidenberg, 1997). It is defined as the strength with which a feature such as $<$ is nutritious $>$ is correlated with the other features of a concept - for example, grapefruit. This variable is calculated by summing the proportion of shared variance between $<$ is nutritious $>$ and each of the other features of grapefruit with which it is significantly correlated. Note that the resulting number is no longer a percentage as such, because it is greater than 100 for some of the concept-feature combinations. The remaining columns are duplicates of many of the variables that are included in the previous two files.

Concept similarities. The cos_matrix_brm_IFR.xls file is a $541 \times 541$ matrix of cosines between concept pairs. To compute this matrix, we took the 541 concepts $X$ 2,526 features matrix and calculated the cosine between each pair of concepts (on the basis of production frequencies). The cosine is calculated as the dot product between two concept vectors, divided by the product of their lengths. The file contains the full matrix, with the main diagonal equal to 1.0 in all cases (the cosine between a concept and itself). Cosine ranges from -1 (opposite vectors) to 1 (identical vectors), with 0 indicating independent vectors. Because Excel files are limited to 256 columns, the matrix is split into three worksheets, with each of the first two including 200 columns and the third including the remaining 141 columns. All three Excel 
worksheets contain all 541 rows, so the similarity of any concept with each of the other concepts is represented in the column labeled by the concept in question.

\section{SUMMARY}

Semantic feature production norms have played and continue to play an important role in the constructing of theories and models of semantic memory and of concepts and categorization, as well as in empirically testing them. This article has described a large set of norms and numerous statistics calculated from them, as well as further descriptive statistics collected from participants' ratings and language corpora. These norms have proven invaluable for a number of projects that we and others have previously conducted, and we hope that they will do so for those who use them in the future.

\section{REFERENCES}

AshCraft, M. H. (1978a). Feature dominance and typicality effects in feature statement verification. Journal of Verbal Learning \& Verbal Behavior, 17, 155-164.

AsHCRAFT, M. H. (1978b). Property norms for typical and atypical items from 17 categories: A description and discussion. Memory \& Cognition, 6, 227-232.

Barsalou, L. W. (1999). Perceptual symbol systems. Behavioral \& Brain Sciences, 22, 577-660.

Barsalou, L. W. (2003). Abstraction in perceptual symbol systems. Philosophical Transactions of the Royal Society of London: Series B, 358, 1177-1187.

Barsalou, L. W., Sloman, S. A., \& Chaigneau, S. E. (2005). The HIPE theory of function. In L. Carlson \& E. van der Zee (Eds.), Representing functional features for language and space: Insights from perception, categorization and development (pp. 131-147). New York: Oxford University Press.

Battig, W. F., \& Montague, W. E. (1969). Category norms for verbal items in 56 categories: A replication and extension of the Connecticut category norms. Journal of Experimental Psychology, 80 (3, Pt. 2), 1-46.

Bourne, L. E., JR., \& Restle, F. (1959). Mathematical theory of concept identification. Psychological Review, 66, 278-296.

Burnard, L. (2000). British National Corpus User Reference Guide Version 2.0. Oxford: Oxford University Computing Service. Retrieved May 20, 2002 from hcu.ox.ac.uk/BNC/World/HTML/urg.html. Data retrieved summer 2001 from http://sara.natcorp.ox.ac.uk.

Collins, A. M., \& LofTus, E. F. (1975). A spreading-activation theory of semantic processing. Psychological Review, 82, 407-428.

Cree, G. S., \& McRaE, K. (2003). Analyzing the factors underlying the structure and computation of the meaning of chipmunk, cherry, chisel, cheese, and cello (and many other such concrete nouns). Journal of Experimental Psychology: General, 132, 163-201.

Cree, G. S., McRae, K., \& McNorgan, C. (1999). An attractor model of lexical conceptual processing: Simulating semantic priming. Cognitive Science, 23, 371-414.

Daugherty, K., \& Seidenberg, M. S. (1992). Rules or connections? The past tense revisited. In Proceedings of the 14th Annual Meeting of the Cognitive Science Society (pp. 259-264). Hillsdale, NJ: Erlbaum.

DAvis, C. J. (2005). N-Watch: A program for deriving neighborhood size and other psycholinguistic statistics. Behavior Research Methods, 37, 65-70.

Devlin, J. T., Gonnerman, L. M., Andersen, E. S., \& Seidenberg, M. S. (1998). Category-specific semantic deficits in focal and widespread brain damage: A computational account. Journal of Cognitive Neuroscience, 10, 77-94.

Garrard, P., Lambon Ralph, M. A., Hodges, J. R., \& Patterson, K. (2001). Prototypicality, distinctiveness, and intercorrelation: Analyses of the semantic attributes of living and nonliving concepts. Cognitive Neuropsychology, 18, 125-174.
Hampton, J. A. (1979). Polymorphous concepts in semantic memory. Journal of Verbal Learning \& Verbal Behavior, 18, 441-461.

Hampton, J. A. (1997). Conceptual combination: Conjunction and negation of natural concepts. Memory \& Cognition, 25, 888-909.

Harm, M., \& SeidenberG, M. S. (2004). Computing the meanings of words in reading: Cooperative division of labor between visual and phonological processes. Psychological Review, 111, 662-720.

Hinton, G. E., \& Shallice, T. (1991). Lesioning an attractor network: Investigations of acquired dyslexia. Psychological Review, 98, 74-95.

Hintzman, D. L. (1986). "Schema abstraction" in a multiple-trace memory model. Psychological Review, 93, 411-428.

Jones, S. S., \& SMith, L. B. (1993). The place of perception in children's concepts. Cognitive Development, 8, 113-139.

KeIL, F. C. (1989). Concepts, kinds, and cognitive development. Cambridge, MA: MIT Press.

KUČERA, H., \& FRANCIS, W. N. (1967). A computational analysis of presentday American English. Providence, RI: Brown University Press.

McNorgan, C., Kotack, R. A., Meehan, D. C., \& McRae, K. (in press). Feature-feature causal relations and statistical co-occurrences in object concepts. Memory \& Cognition.

McRAe, K. (2004). Semantic memory: Some insights from featurebased connectionist attractor networks. In B. H. Ross (Ed.), Psychology of learning and motivation (Vol. 45, pp. 41-86). San Diego: Academic Press.

McRae, K., Cree, G. S., Cho, M. J., \& McNorgan, C. (2003, September). Distinguishing knowledge of living and nonliving things is computed quickly from concept names, and vice versa. Poster presented at the Thirteenth Conference of the European Society for Cognitive Psychology, Granada, Spain.

McRae, K., Cree, G. S., Westmacott, R., \& de SA, V. R. (1999). Further evidence for feature correlations in semantic memory. Canadian Journal of Experimental Psychology, 53, 360-373.

McRae, K., De SA, V. R., \& Seidenberg, M. S. (1997). On the nature and scope of featural representations of word meaning. Journal of Experimental Psychology: General, 126, 99-130.

McRae, K., Ferretti, T. R., \& Amyote, L. (1997). Thematic roles as verbspecific concepts. Language \& Cognitive Processes, 12, 137-176.

Medin, D. L. (1989). Concepts and conceptual structure. American Psychologist, 44, 1469-1481.

Medin, D. L., \& Schaffer, M. M. (1978). Context theory of classification learning. Psychological Review, 85, 207-238.

Medin, D. L., \& Shoben, E. J. (1988). Context and structure in conceptual combination. Cognitive Psychology, 20, 158-190.

Minda, J. P., \& Smith, J. D. (2002). Comparing prototype-based and exemplar-based accounts of category learning and attentional allocation. Journal of Experimental Psychology: Learning, Memory, \& Cognition, 28, 275-292.

Moss, H. E., Tyler, L. K., \& Devlin, J. T. (2002). The emergence of category-specific deficits in a distributed semantic system. In E. M. E. Forde \& G. W. Humphreys (Eds.), Category-specificity in brain and mind (pp. 115-147). East Sussex, U.K.: Psychology Press.

Murdock, B. B. (1982). A theory for the storage and retrieval of item and associative information. Psychological Review, 89, 609-626.

Pecher, D., Zeelenberg, R., \& Barsalou, L. W. (2003). Verifying different-modality properties for concepts produces switching costs. Psychological Science, 14, 119-124.

Pexman, P. M., Holyk, G. G., \& Monfils, M.-H. (2003). Number-offeatures effects and semantic processing. Memory \& Cognition, 31, 842-855.

Pexman, P. M., Lupker, S. J., \& Hino, Y. (2002). The impact of feedback semantics in visual word recognition: Number-of-features effects in lexical decision and naming tasks. Psychonomic Bulletin \& Review, 9, 542-549.

Plaut, D. C. (2002). Graded modality-specific specialization in semantics: A computational account of optic aphasia. Cognitive Neuropsychology, 19, 603-639.

Plaut, D. C., \& Shallice, T. (1993). Deep dyslexia: A case study of connectionist neuropsychology. Cognitive Neuropsychology, 10, 377-500.

Rogers, T. T., \& McClelland, J. L. (2004). Semantic cognition: A parallel distributed processing approach. Cambridge, MA: MIT Press.

Rosch, E., \& Mervis, C. B. (1975). Family resemblances: Studies in the internal structure of categories. Cognitive Psychology, 7, 573-605. 
Smith, E. E., \& Medin, D. L. (1981). Categories and concepts. Cambridge, MA: Harvard University Press.

Smith, E. E., Osherson, D. N., Rips, L. J., \& Keane, M. (1988). Combining prototypes: A selective modification model. Cognitive Science, 12, 485-527.

Smith, E. E., Shoben, E. J., \& RiPS, L. J. (1974). Structure and process in semantic memory: A feature model for semantic decisions. Psychological Review, 81, 214-241.

Solomon, K. O., \& Barsalou, L. W. (2001). Representing properties locally. Cognitive Psychology, 43, 129-169.

Tversky, A. (1977). Features of similarity. Psychological Review, 84, 327-352.

Vigliocco, G., Vinson, D. P., Lewis, W., \& Garrett, M. F. (2004). Representing the meaning of object and action words: The featural and unitary semantic space (FUSS) hypothesis. Cognitive Psychology, 48, 422-488.

VINSON, D. P., \& VigLIOCCO, G. (2002). A semantic analysis of noun-verb dissociations in aphasia. Journal of Neurolinguistics, 15, 317-351.

Wu, L. L., \& Barsalou, L. W. (2004). Grounding concepts in perceptual simulation: 1. Evidence from property generation. Manuscript under review.

\section{ARCHIVED MATERIALS}

The following materials and links can be accessed through the Psychonomic Society's Norms, Stimuli, and Data archive, http://www .psychonomic.org/archive/.

To access these files or links, search the archive for this article using the journal (Behavior Research Methods), the first author's name (McRae), and the publication year (2005).

FILE: McRae-BRM-2005.zip.
DESCRIPTION: The compressed archive file expands into a directory called McRae-BRM-2005, which includes nine data files, plus two instruction files.

README.txt provides instructions on how to reconstruct the original 4 files in Excel. The files were constructed originally in Excel, and each consisted of at least two worksheets. The files are:

CONCS_brm.txt, a $64 \mathrm{~K}$ file that contains information on individual concepts.

CONCS_brm_variable_explanations.txt, a $4 \mathrm{~K}$ file that defines each column in CONCS_brm.txt.

CONCS_FEATS_concstats_brm.txt, a 1.3-MB file that contains information on all features in all concepts.

CONCS_FEATS_concstats_brm_variable_explanations.txt, a $4 \mathrm{~K}$ file that defines each column in CONCS_FEATS_concstats_brm.txt.

FEATS_brm.txt, a 156K file that contains information on individual features.

FEATS_brm_variable_explanations.txt, a $4 \mathrm{~K}$ file that defines each column in FEATS_brm.txt.

cos_matrix_brm_IFR_1-200.txt, a 640K file that contains similarities measured via cosine between each of the 541 concepts (rows in matrix) and the first 200 concepts (columns).

cos_matrix_brm_IFR_201-400.txt, a 640K file that contains similarities measured via cosine between each of the 541 concepts (rows in matrix) and the second 200 concepts (columns).

cos_matrix_brm_IFR_401-541.txt, a 456K file that contains the similarity in terms of cosine between each of the 541 concepts (rows in matrix) and the last 141 concepts (columns).

AUTHOR's E-MAIL ADDRESS: mcrae@uwo.ca

AutHOR's WeB SITE: http://www.ssc.uwo.ca/psychology/faculty/ mcrae_res.htm.

APPENDIX A

Featural Representations for Moose and Knife

\begin{tabular}{clcl}
\hline $\begin{array}{c}\text { Concept } \\
\text { Name }\end{array}$ & \multicolumn{1}{c}{ Feature } & $\begin{array}{c}\text { Production } \\
\text { Frequency }\end{array}$ & \multicolumn{1}{c}{ Brain Region } \\
Moose & is large & 27 & visual-form and surface \\
& has antlers & 23 & visual-form and surface \\
& has legs & 14 & visual-form and surface \\
& has four legs & 12 & visual-form and surface \\
& has fur & 7 & visual-form and surface \\
& has hair & 5 & visual-form and surface \\
& has hooves & 5 & visual-form and surface \\
& is brown & 10 & visual-color \\
& hunted by people & 17 & function \\
& eaten as meat & 5 & function \\
& lives in woods & 14 & encyclopedic \\
& lives in wilderness & 8 & encyclopedic \\
& an animal & 17 & taxonomic \\
a mammal & 9 & taxonomic \\
an herbivore & 8 & taxonomic \\
Knife & has a handle & 14 & visual-form and surface \\
& has a blade & 11 & visual-form and surface \\
made of steel & 8 & visual-form and surface \\
made of metal & 7 & visual-form and surface \\
made of stainless steel & 5 & visual-form and surface \\
is shiny & 5 & visual-form and surface \\
used for cutting & 25 & function \\
used for killing & 7 & function \\
used by butchers & 5 & function \\
is sharp & 29 & tactile \\
is serrated & 8 & tactile \\
& & & \\
& & &
\end{tabular}




\begin{tabular}{llcl}
\multicolumn{4}{c}{ APPENDIX A (Continued) } \\
\hline $\begin{array}{llcl}\text { Concept } \\
\text { Name }\end{array}$ & \multicolumn{1}{c}{ Feature } & Production & \multicolumn{1}{c}{ Brain Region } \\
\hline Knife & Frequency & Classification \\
& is dangerous & 14 & encyclopedic \\
& found in kitchens & 8 & encyclopedic \\
& used with forks & 6 & encyclopedic \\
& a weapon & 11 & taxonomic \\
& a utensil & 9 & taxonomic \\
& a cutlery & 5 & taxonomic \\
\hline
\end{tabular}

\section{APPENDIX B
Instructions for Feature Production Norms}

This experiment is part of an investigation into how people read words for meaning. To help us conduct this work, we need information on what people know about different things in the world. On the following pages, there are words that each denote a concept, with each being followed by 14 blank lines. Please fill in as many of these lines as you can with properties of the concept to which the word refers. Examples of different types of properties would be: physical properties, such as internal and external parts, and how it looks, sounds, smells, feels, or tastes; functional properties, such as what it is used for; where, when and by whom it is used; things that the concept is related to, such as the category that it belongs in; and other facts, such as how it behaves, or where it comes from. Please note that even though many of the words can be thought of as something other than a noun (e.g., "camp" can refer to the place where your tent is pitched, or the action of camping), all words on the following pages are meant to be considered as nouns only (e.g., "camp," the place). Below, we have provided 3 examples to give you an idea of what might be considered a property description of a concept.

\section{duck}

is a bird

is an animal

waddles

flies

migrates

lays eggs

quacks

swims

has wings

has a beak

has webbed feet

has feathers

lives in ponds

lives in water

hunted by people

is edible cucumber

is a vegetable

has green skin

has a white inside

has seeds inside

is cylindrical

is long

grows in gardens

grows on vines

is edible

is crunchy

used for making pickles

eaten in salads stove

is an appliance produces heat has elements has an oven made of metal is hot is electrical runs on wood runs on gas found in kitchens used for baking used for cooking food

You may be able to think of more and/or different types of properties for these concepts, but these examples should give you an idea of what is requested of you. Please do not languish an extraordinary amount of time on each word, but also please take a bit of time to consider the relevant properties of each entity or object. In other words, complete this questionnaire reasonably quickly, but keep the relevant types of properties in mind. Thank you very much for completing this questionnaire.

APPENDIX C

Explanations of Variables Included in the Concepts File

\begin{tabular}{ll}
\hline \multicolumn{1}{c}{ Variable } & \multicolumn{1}{c}{ Explanation } \\
\hline Concept & concept name \\
Pronunciation & pronunciation according to Neighborhood Watch program \\
Phon_1st & initial phoneme of concept name \\
KF & Kučera and Francis (1967) frequency: sum of singular and plural \\
$\ln (\mathrm{KF})$ & natural logarithm of Kučera and Francis (1967) frequency: sum of singular \\
& and plural \\
BNC & British National Corpus frequency: sum of singular and plural \\
$\ln (\mathrm{BNC})$ & natural logarithm of: British National Corpus frequency: sum of singular and \\
& plural \\
Familiarity & mean rated concept familiarity by 20 participants: $1-9$ scale, $9=$ extremely \\
& familiar
\end{tabular}


APPENDIX C (Continued)

\begin{tabular}{|c|c|}
\hline Variable & Explanation \\
\hline Length_Letters & number of letters in concept name \\
\hline Length_Phonemes & number of phonemes in concept name \\
\hline Length_Syllables & number of syllables in concept name \\
\hline Bigram & $\begin{array}{l}\text { mean position-specific bigram frequency according to Neighborhood Watch } \\
\text { program }\end{array}$ \\
\hline Trigram & $\begin{array}{l}\text { mean position-specific trigram frequency according to Neighborhood Watch } \\
\text { program }\end{array}$ \\
\hline ColtheartN & Coltheart's $N$ according to Neighborhood Watch program \\
\hline Num_Feats_Tax & number of features, including taxonomic features \\
\hline Num_Feats_No_Tax & number of features, excluding taxonomic features \\
\hline Num_Disting_Feats_No_Tax & $\begin{array}{l}\text { number of distinguishing features (those in }<3 \text { concepts in norms), excluding } \\
\text { taxonomic features }\end{array}$ \\
\hline Disting_Feats_\%_No_Tax & $\begin{array}{l}\text { percentage of features that are distinguishing features (those in }<3 \text { concepts in } \\
\text { norms), excluding taxonomic features }\end{array}$ \\
\hline Mean_Distinct_No_Tax & $\begin{array}{l}\text { mean distinctiveness of the concept's features ( } 1 \text { /number of concepts in which } \\
\text { feature occurs), excluding taxonomics }\end{array}$ \\
\hline Mean_CV_No_Tax & mean cue validity of the concept's features, excluding taxonomics \\
\hline Density_No_Tax & $\begin{array}{l}\text { intercorrelational density: sum of } r^{2} \text { for the concept's significantly correlated } \\
\text { feature pairs }\end{array}$ \\
\hline $\begin{array}{l}\text { Num_Corred_Pairs_No_Tax } \\
\% \text { _Corred_Pairs_No_Tax }\end{array}$ & $\begin{array}{l}\text { number of significantly correlated feature pairs, excluding taxonomics } \\
\text { percentage of possible feature pairs that are correlated, excluding taxonomics }\end{array}$ \\
\hline \multicolumn{2}{|c|}{ The remaining are based on Cree and McRae's (2003) brain region taxonomy. } \\
\hline Num_Func & number of functional features in concept \\
\hline Num_Vis_Mot & number of visual-motor features in concept \\
\hline Num_Vis_F\&S & number of visual form and surface features in concept \\
\hline Num_Vis_Col & number of visual color features in concept \\
\hline Num_Sound & number of sound features in concept \\
\hline Num_Taste & number of taste features in concept \\
\hline Num_Smell & number of smell features in concept \\
\hline Num_Tact & number of tactile features in concept \\
\hline Num_Ency & number of encyclopedic features in concept \\
\hline Num_Tax & number of taxonomic features in concept \\
\hline
\end{tabular}

APPENDIX D

Explanations of Variables Included in the Features File

\begin{tabular}{|c|c|}
\hline Variable & Explanation \\
\hline Feature & feature name \\
\hline $\mathrm{CPF}$ & number of concepts for which the feature was listed by at least 5 of 30 subjects \\
\hline Disting & $\begin{array}{l}\mathrm{D}=\text { distinguishing (listed for } 1 \text { or } 2 \text { concepts in norms), ND = nondistin- } \\
\text { guishing }(>2 \text { concepts })\end{array}$ \\
\hline Distinct & $\begin{array}{l}\text { distinctiveness }=1 / \mathrm{cpf} \text { : the inverse of the number of concepts in which that } \\
\text { feature occurs }\end{array}$ \\
\hline Feat_Length_Including_Spaces & number of characters in feature name, including the spaces as characters \\
\hline BR_Label & feature type based on Cree and McRae's (2003) brain region taxonomy \\
\hline
\end{tabular}

APPENDIX E

Explanations of Variables Included in the Concepts-Features File

\begin{tabular}{ll}
\hline \multicolumn{1}{c}{ Variable } & \multicolumn{1}{c}{ Explanation } \\
\hline Concept & concept name \\
Feature & feature name \\
WB_Label & feature type according to the taxonomy of Wu and Barsalou (2004) \\
WB_Maj & feature type major classification according to the taxonomy of Wu and Bar- \\
& salou (2004) \\
WB_Min & feature type minor classification according to the taxonomy of Wu and Bar- \\
& salou (2004)
\end{tabular}


APPENDIX E (Continued)

\begin{tabular}{|c|c|}
\hline Variable & Explanation \\
\hline BR_Label & feature type based on Cree and McRae's (2003) brain region taxonomy \\
\hline Prod_Freq & $\begin{array}{l}\text { production frequency: the number of participants out of a possible } 30 \text { who } \\
\text { listed that feature for that concept in the norms }\end{array}$ \\
\hline Rank_PF & the feature's rank within the concept on the basis of production frequency \\
\hline Sum_PF_No_Tax & $\begin{array}{l}\text { sum of the production frequencies for that feature across all concepts in which } \\
\text { it occurs, excluding taxonomic features }\end{array}$ \\
\hline $\mathrm{CPF}$ & the number of concepts in which that feature occurs \\
\hline Disting & $\begin{array}{l}\mathrm{D}=\text { distinguishing feature, } \mathrm{ND}=\text { nondistinguishing feature, where distin- } \\
\text { guishing is defined as occurring in one or two concepts in the norms }\end{array}$ \\
\hline Distinct & $\begin{array}{l}\text { distinctiveness }=1 / \mathrm{cpf} \text { : the inverse of the number of concepts in which that } \\
\text { feature occurs }\end{array}$ \\
\hline CV_No_Tax & $\begin{array}{l}\text { cue validity of the feature for that concept, excluding all taxonomic features; } \\
\text { CV }=\text { (Prod_Freq)/Sum_PF_No_Tax }\end{array}$ \\
\hline Intercorr_Str_Tax & $\begin{array}{l}\text { intercorrelational strength of the feature for that concept, includes taxonomic } \\
\text { features }\end{array}$ \\
\hline Intercorr_Str_No_Tax & $\begin{array}{l}\text { intercorrelational strength of feature for that concept, excludes taxonomic } \\
\text { features }\end{array}$ \\
\hline Feat_Length_Including_Spaces & number of characters in feature name, including the spaces as characters \\
\hline Phon_1st & initial phoneme of concept name \\
\hline $\mathrm{KF}$ & $\begin{array}{l}\text { Kučera and Francis (1967) frequency of concept name: sum of singular and } \\
\text { plural }\end{array}$ \\
\hline $\ln (\mathrm{KF})$ & $\begin{array}{l}\text { natural logarithm of Kučera and Francis (1967) frequency: sum of singular } \\
\text { and plural }\end{array}$ \\
\hline $\mathrm{BNC}$ & British National Corpus frequency of concept name: sum of singular and plural \\
\hline $\ln (\mathrm{BNC})$ & $\begin{array}{l}\text { natural logarithm of British National Corpus frequency of concept name: sum } \\
\text { of singular and plural }\end{array}$ \\
\hline Familiarity & $\begin{array}{l}\text { mean rated concept familiarity by } 20 \text { participants: } 1-9 \text { scale, } 9=\text { extremely } \\
\text { familiar }\end{array}$ \\
\hline Length_Letters & number of letters in concept name \\
\hline Length_Phonemes & number of phonemes in concept name \\
\hline Length_Syllables & number of syllables in concept name \\
\hline Bigram & $\begin{array}{l}\text { mean position-specific bigram frequency of concept name according to } \\
\text { Neighborhood Watch program }\end{array}$ \\
\hline Trigram & $\begin{array}{l}\text { mean position-specific trigram frequency of concept name according to } \\
\text { Neighborhood Watch program }\end{array}$ \\
\hline ColtheartN & Coltheart's $N$ of concept name according to Neighborhood Watch program \\
\hline Num_Feats_Tax & number of features in concept, including taxonomic features \\
\hline Num_Feats_No_Tax & number of features in concept, excluding taxonomic features \\
\hline Num_Disting_Feats_No_Tax & $\begin{array}{l}\text { number of distinguishing features in concept (those in }<3 \text { concepts in norms), } \\
\text { excluding taxonomic features }\end{array}$ \\
\hline Disting_Feats_\%_No_Tax & $\begin{array}{l}\text { percentage of features in concept that are distinguishing features (those in }<3 \\
\text { concepts in norms), excluding taxonomic features }\end{array}$ \\
\hline Mean_Distinct_No_Tax & $\begin{array}{l}\text { mean distinctiveness of the concept's features ( } 1 / \text { number of concepts in which } \\
\text { feature occurs), excluding taxonomics }\end{array}$ \\
\hline Mean_CV_No_Tax & mean cue validity of the concept's features, excluding taxonomics \\
\hline Density_No_Tax & $\begin{array}{l}\text { intercorrelational density of the concept: sum of } r^{2} \text { for the concept's signifi- } \\
\text { cantly correlated feature pairs }\end{array}$ \\
\hline Num_Corred_Pairs_No_Tax & $\begin{array}{l}\text { number of significantly correlated feature pairs in concept, excluding taxo- } \\
\text { nomics }\end{array}$ \\
\hline$\%$ \%Corred_Pairs_No_Tax & $\begin{array}{l}\text { percentage of possible feature pairs in concept that are correlated, excluding } \\
\text { taxonomics }\end{array}$ \\
\hline Num_Func & number of functional features in concept \\
\hline Num_Vis_Mot & number of visual-motor features in concept \\
\hline Num_VisF\&S & number of visual form and surface features in concept \\
\hline Num_Vis_Col & number of visual color features in concept \\
\hline Num_Sound & number of sound features in concept \\
\hline Num_Taste & number of taste features in concept \\
\hline Num_Smell & number of smell features in concept \\
\hline Num_Tact & number of tactile features in concept \\
\hline Num_Ency & number of encyclopedic features in concept \\
\hline Num_Tax & number of taxonomic features in concept \\
\hline
\end{tabular}


Example of Each Wu and Barsalou (2004) Feature Type Listed for the 541 Concepts

\begin{tabular}{|c|c|c|c|}
\hline Class & Feature Type & $n$ & Example \\
\hline Entity & $\begin{array}{l}\text { Associated abstract entity } \\
\text { Entity behavior } \\
\text { External component } \\
\text { External surface property } \\
\text { Internal component } \\
\text { Internal surface property } \\
\text { Larger whole } \\
\text { Made-of } \\
\text { Quantity } \\
\text { Systemic feature }\end{array}$ & $\begin{array}{r}19 \\
525 \\
1,084 \\
1,179 \\
192 \\
178 \\
15 \\
476 \\
14 \\
298\end{array}$ & $\begin{array}{l}\text { harp }<\text { associated with angels }> \\
\text { clock }<\text { ticks }> \\
\text { tricycle }<\text { has pedals }> \\
\text { apple }<\text { is red }> \\
\text { cherry }<\text { has a pit }> \\
\text { fridge }<\text { is cold }> \\
\text { ant }<\text { lives in a colony }> \\
\text { sink }<\text { made of enamel }> \\
\text { slippers }<\text { come in pairs }> \\
\text { dolphin }<\text { is intelligent }>\end{array}$ \\
\hline Situation & $\begin{array}{l}\text { Action/manner } \\
\text { Associated entity } \\
\text { Function } \\
\text { Location } \\
\text { Origin } \\
\text { Participant } \\
\text { Time }\end{array}$ & $\begin{array}{r}186 \\
158 \\
1,145 \\
466 \\
64 \\
187 \\
85\end{array}$ & $\begin{array}{l}\text { screws }<\text { used by turning }> \\
\text { saucer }<\text { used with tea cups }> \\
\text { tomato }<\text { eaten }> \\
\text { cupboard }<\text { found in kitchens }> \\
\text { walnut }<\text { grows on trees }> \\
\text { wand }<\text { used by magicians }> \\
\text { turkey }<\text { eaten at Thanksgiving }>\end{array}$ \\
\hline Taxonomic & $\begin{array}{l}\text { Coordinate } \\
\text { Individual } \\
\text { Subordinate } \\
\text { Superordinate } \\
\text { Synonym }\end{array}$ & $\begin{array}{r}52 \\
3 \\
45 \\
601 \\
29\end{array}$ & $\begin{array}{l}\text { coyote }<\mathrm{dog}> \\
\text { deer }<\text { Bambi }> \\
\text { lettuce }<\text { romaine }> \\
\text { deer }<\text { a mammal }> \\
\text { calf }<\text { baby cow }>\end{array}$ \\
\hline Introspective & $\begin{array}{l}\text { Affect emotion } \\
\text { Cognitive operation } \\
\text { Contingency } \\
\text { Evaluation } \\
\text { Negation }\end{array}$ & $\begin{array}{r}11 \\
30 \\
93 \\
105 \\
19\end{array}$ & $\begin{array}{l}\text { wasp }<\text { is annoying }> \\
\text { magazine }<\text { like a book }> \\
\text { garlic }<\text { causes bad breath }> \\
\text { gown }<\text { is fancy }> \\
\text { ostrich }<\text { cannot fly }>\end{array}$ \\
\hline Total & & 7,259 & \\
\hline
\end{tabular}

(Manuscript received October 7, 2003;

revision accepted for publication September 10, 2004.) 